\title{
Separative Determination of Ascorbic Acid Analogs Contained in Mushrooms by High-Performance Liquid Chromatography
}

\author{
Masato OKAMURA \\ Laboratory of Biochemistry and Nutrition, Kanagawa Prefectural \\ College of Nursing and Medical Technology, \\ Yokohama 241-0815, Japan
}

(Received August 18, 1997)

\begin{abstract}
Summary Analogs (6-deoxyascorbic acid, erythroascorbic acid, and associated glycosides) of L-ascorbic acid (AA) contained in mushrooms were allowed to react with hydrazine to form osazones, and the conditions for separative determination by HPLC using a Zorbax SIL column were examined. Separation was started using solvent system 1 (ethylacetate $/ n$ hexane/acetone/acetic acid, $50: 50: 1: 1, \mathrm{v} / \mathrm{v}$ ) as the mobile phase, and switching after $15 \mathrm{~min}$ to solvent system 2 (ethylacetate/acetone/acetic acid, $100: 1: 1, \mathrm{v} / \mathrm{v})$. Detection was performed by absorbance at $500 \mathrm{~nm}$. Because these analogs showed different formation rates for osazone, calibration curves were prepared for each substance. The recovery rate in the load test was $93-105 \%$. By this method, AA and the analogs contained in eight species of edible mushrooms have been determined. The results revealed that: (1) the main constituents of all mushrooms are AA analogs rather than AA itself; (2) only one species contained AA in a very small amount $(2 \mu \mathrm{mol} / \mathrm{kg}) ;(3)$ the types of AA analogs present differed according to the species of mushrooms, and (4) the total amount of AA analogs was between ca. $100-500 \mu \mathrm{mol} / \mathrm{kg}$ (2-9 mg per $100 \mathrm{~g}$, converted to AA). In addition, a new AA analog was found in Pleurotus ostreatus and identified as 5-O-( $\alpha$-D-xylopyranosyl)-D-erythroascorbic acid in structural analyses by NMR and other methods.
\end{abstract}

Key Words separative determination, ascorbic acid, ascorbic acid analog, mushroom, high-performance liquid chromatography

A previous study ( 1 ) revealed that mushrooms (fruit bodies of Basidiomycotina) contain 6-deoxyascorbic acid (DAA) and erythroascorbic acid (EAA), which are analogs of L-ascorbic acid (AA), as well as their glycosides. The glycosides are 6-deoxy-5-O-( $\alpha$-D-xylopyranosyl)-ascorbic acid (DAA-Xy), 6-deoxy-5-O-( $\alpha$-D-glucopyranosyl)-ascorbic acid (DAA-Glu) and 5-O-( $\alpha$-D-glucopyranosyl)-erythroascorbic acid (EAA-Glu), in which sugar is combined with the C-5 of the analogs. Because 
they have equivalent reducing power, their total amounts can be measured by the $\alpha, \alpha^{\prime}$-dipyridyl method (2). However, there was no practical method for separative determination, and thus the content and distribution of each substance and the existence of AA have not been clarified. The predicted compound of EAA and xylose has also not been found. In addition, other AA analogs are believed to exist in mushrooms, and therefore the development of separative determination is urgently required for their detection.

As separative determinations of AA-related substances, those of AA and D-araboascorbic acid (erythorbic acid) (3, 4), AA, saccharoascorbic acid and AA-2-sulfate (5), and AA and EAA (6) were reported; each of them identified through the use of the high-performance liquid chromatography (HPLC) method. The HPLC method is considered suitable for the separative determination of AA analogs contained in mushrooms, but the confirmation of unknown peaks as new analogs will be difficult with detection by UV absorption measurement or electrochemical methods using the above method. However, an alternative determination method has been reported, in which the oxidized form of AA is allowed to react with 2,4-dinitrophenylhydrazine (hydrazine) to form 2,3-bisdinitrophenylhydrazone (osazone) by the Roe method (7). This osazone was then eluted in a Nucleosil silica column, Bondapak- $\mathrm{NH}_{2}$ column or GL-Pack Partisil-5 column, for analysis $(8-10)$. All AA analogs contained in mushrooms have a five-membered lactone ring, as does AA, and their oxidized form makes osazones. Consequently, it is believed that their separation by HPLC enables the determination of AA analogs, and that when an unknown peak is observed, the measurement of its absorption spectrum makes it easy to determine whether it is a new AA analog. However, methods formerly reported have all been intended to determine AA alone, so they are not suitable for samples such as mushrooms that contain many AA analogs. Therefore, the conditions for separative determination using. HPLC of the AA analogs to turn them into osazones are discussed here, and the resulting satisfactory method is presented. In addition, the determination of eight species of mushrooms, commonly eaten in Japan, through the use of this method revealed that a new AA analog is contained in Pleurotus ostreatus, and its structure was analyzed by nuclear magnetic resonancing (NMR) and other methods.

In this paper, DAA, EAA and associated glycosides are generically referred to as mush-ascorbic acid (mush-AA).

\section{MATERIALS AND METHODS}

Reagents. AA and D-araboascorbic acid were purchased from Tokyo Chemicals Industry. DAA-Xy, DAA-Glu and EAA-Glu were extracted from Flammulina velutipes, Grifola frondosa and Hypsizigus marmoreus, respectively, and purified by the method described in a previous paper (1). DAA was prepared as follows: DAA-Xy was dissolved in $3 \mathrm{~N} \mathrm{HCl}$, heated in a boiling water bath for $15 \mathrm{~min}$ for hydrolysis and purified. EAA was obtained from EAA-Glu in a similar 
manner as above. Other reagents used for analysis were commercially available in chromatograph grade, and were of the highest quality.

Preparation of the mushroom extract. Ten grams of fresh mushrooms were ground with $20 \mathrm{~mL}$ of ethanol cooled to $-20^{\circ} \mathrm{C}$ and filtered after leaving in ice water for $10 \mathrm{~min}$ to obtain the extract. All apparatuses used for grinding were cooled to $-20^{\circ} \mathrm{C}$ in advance.

Formation of osazones. A $0.5 \mathrm{~mL}$ volume of $25 \% \mathrm{HPO}_{3}$ was added to $2 \mathrm{~mL}$ of the mushroom extract, and then saturated 2,6-dichlorophenolindophenol (indophenol) solution was added dropwise until AA and its analogs turned into oxidized form with pink color remaining. After $30 \mathrm{~s}, 0.1 \mathrm{~mL}$ of $10 \%$ thiourea and $0.4 \mathrm{~mL}$ of $2 \%$ hydrazine (dissolved in $25 \%$ sulfuric acid) were added and incubated for $3 \mathrm{~h}$ at $37^{\circ} \mathrm{C}$. Then, $6 \mathrm{~mL}$ of ethylacetate was added, the mixture was shaken vigorously, and the reactant and unreacted hydrazine were extracted. The ethylacetate layer was pipetted and $1 \mathrm{~g}$ of $\mathrm{Na}_{2} \mathrm{SO}_{4}$ was added to remove water. After drying $3 \mathrm{~mL}$ of the dehydrated ethylacetate layer in vacuo, the residue was dissolved in $3 \mathrm{~mL}$ of solvent system 1 (ethylacetate $/ n$-hexane/acetone/acetic acid, $50: 50: 1: 1, \mathrm{v} / \mathrm{v}$ ) and used for HPLC analysis.

Measurement of absorption spectrum of osazones. Each mush-AA was allowed to form osazone, which was then extracted with ethylacetate and purified by developing on thin-layer chromatography (TLC) using Wakogel FM Plate (Wako Pure Chemicals Co.) with toluene/acetone/acetic acid $(2: 1: 1, \mathrm{v} / \mathrm{v})$ as the developing solvent. Each purified substance was dissolved in solvent system 2 (ethylacetate/ acetone/acetic acid, 100:1:1, v/v), and its absorption spectrum was measured using a 100-10 spectrophotometer (Hitachi Co.).

HPLC. The HPLC system consisted of a Shimadzu LC-10AS liquid chromatographer (Shimadzu Co.), a Rheodyne injection valve with a $20 \mathrm{~mL}$ sample loop, a Shimadzu SPD-10A UV-Vis spectrophotometric detector set at $500 \mathrm{~nm}$ and a Shimadzu Chromatopac C-R 5A. The column used was Zorbax SIL $4.6 \mathrm{~mm} \times 25 \mathrm{~cm}$ (Du Pont Co.). Separation was started using solvent system 1 as the mobile phase, and switching after $15 \mathrm{~min}$ to solvent system 2 . The flow rate was $1 \mathrm{~mL} / \mathrm{min}$.

Structural analysis of newly discovered AA analog. Structural analysis was performed using the ultraviolet spectrum, mass spectrum (MS) and NMR spectrum. The apparatuses used and the measurement conditions are shown in the results section.

\section{RESULTS}

Comparison of the osazone formation rates of mush-AA and osazone absorption spectrum

Samples of AA, EAA, EAA-Glu, DAA, DAA-Xy and DAA-Glu (50 $\mu \mathrm{M}$ each) were oxidized by indophenol, and hydrazine was added to each of them and incubated at $37^{\circ} \mathrm{C}$. Two milliliters of reaction liquid was removed at predetermined intervals, and an equal amount of $85 \%$ sulfuric acid was added to dissolve the 
osazones. The formation of osazone was then monitored by measuring absorbance at $530 \mathrm{~nm}$. The amount of osazone formation by mush-AA was lower than that of AA, and those of DAA and its glycosides were particularly low. Neither by prolonging the reaction time nor changing the reaction temperature could conditions be created under which all mush-AA formed osazone as AA. Therefore, in consideration of the operation procedures, the incubating time was fixed at $3 \mathrm{~h}$ at $37^{\circ} \mathrm{C}$. Under these conditions, when the osazone formation rate of AA was 1 , the rates of the other samples were as follows: DAA, 0.50; DAA-Xy, 0.38; DAA-Glu, 0.30; EAA, 0.95; and EAA-Glu, 0.90.

Absorption spectra in the visible field of all mush-AA-osazones were consistent with that of AA-osazone previously well known, and the absorption maxima dissolved in the solvent system 2 were all in the vicinity of $500 \mathrm{~nm}$.

\section{HPLC analysis of AA-osazone and mush-AA-osazones}

A solution containing $50 \mu \mathrm{M}$ each of AA, DAA, EAA, DAA-Xy, DAA-Glu and EAA-Glu was used as a sample. Osazones were formed, and were determined by HPLC using a Zorbax SIL column. A study of the mobile phase revealed that the peaks of the target substances favorably separated by using solvent system 1 for the first $15 \mathrm{~min}$, and then solvent system 2 later (Fig. 1). The retention times of the osazones $\left(t_{\mathrm{R}}, \mathrm{min}\right)$ were as follows: DAA, 5.6; EAA, 8.4; AA, 16.0; DAA-Xy, 23.3; DAA-Glu, 26.3 and EAA-Glu, 29.2.

Organisms, such as Penicillium, that synthesize D-araboascorbic acid were

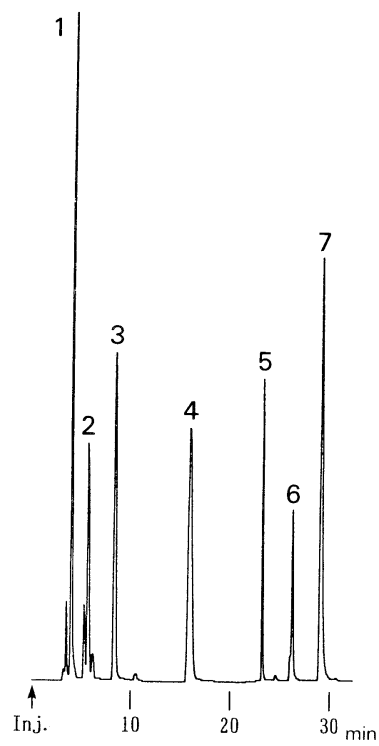

Fig. 1. HPLC profiles of AA-osazone and mush-AA-osazones. Sample peaks: 1, hydrazine; 2, DAA; 3, EAA; 4, AA; 5, DAA-Xy; 6, DAA-Glu; 7, EAA-Glu. 


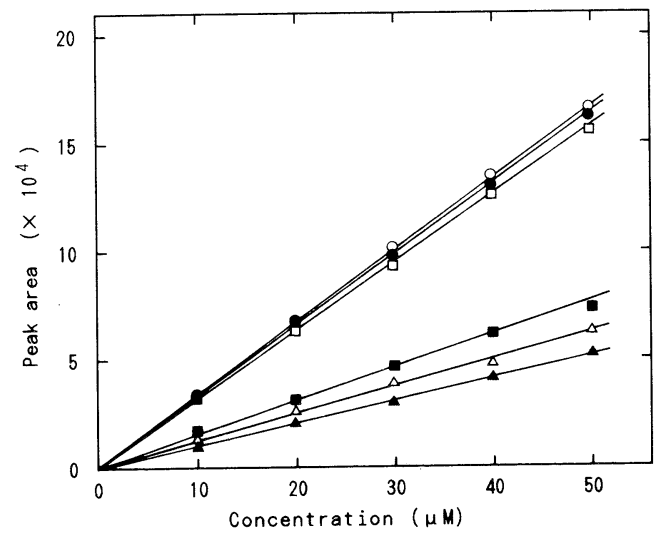

Fig. 2. The calibration curves of AA (O), EAA (๑), EAA-Glu ( $\square$ ), DAA ( $\square$ ), DAA-Xy $(\triangle)$ and DAA-Glu $(\boldsymbol{\Delta})$ for chromatographic analysis.

reported (11), but the $t_{\mathrm{R}}$ of $\mathrm{D}$-araboascorbic acid osazone is 17.8 , so it is distinguishable from AA.

\section{Calibration curves}

The formation ratios of the osazones of each mush-AA vary, so calibration curves were prepared for each substance. The calibration curves by peak area were all linear, passing through the origin in the range of $0-50 \mu \mathrm{M}$ (Fig. 2). Measured values showed some dispersion and the standard deviation was $\pm 5.5 \%$. The lower limit of accurate measurement was $0.1 \mu \mathrm{M}$ for AA and $0.4 \mu \mathrm{M}$ for DAA-Glu, which had the lowest osazone formation rate.

\section{Recovery experiments for $A A$ and mush- $A A$}

The extract obtained by grinding $10 \mathrm{~g}$ of Flammulina velutipes in $20 \mathrm{~mL}$ of ethanol containing $67 \mu \mathrm{M}$ each of AA, DAA, EAA, DAA-Xy, DAA-Glu and EAA-Glu was used as a specimen to form osazones, and was analyzed by HPLC. The experiment was also carried out with a ground specimen in ethanol containing no additional AA or mush-AA, and the recovery rates of AA and mush-AA supplements were determined by the difference between these two specimens. The recovery rates $(n=10)$ were as follows: AA, $98 \pm 4 \%$; DAA, $95 \pm 7 \%$; EAA, $93 \pm 2 \%$; DAA-Xy, $98 \pm 5 \%$; DAA-Glu, $105 \pm 8 \%$ and EAA-Glu, $100 \pm 3 \%$. The recovery rate for EAA is rather low, though it would be a satisfactory value for the method employed for ordinary measurements.

\section{Measurement of the AA and mush-AA contents of mushrooms}

The AA and mush-AA contained in eight species of fresh edible mushrooms were determined by this method (Table 1). Pleurotus ostreatus contains DAA, EAA and all three kinds of glycoside (DAA-Xy, DAA-Glu and EAA-Glu). In addition, 


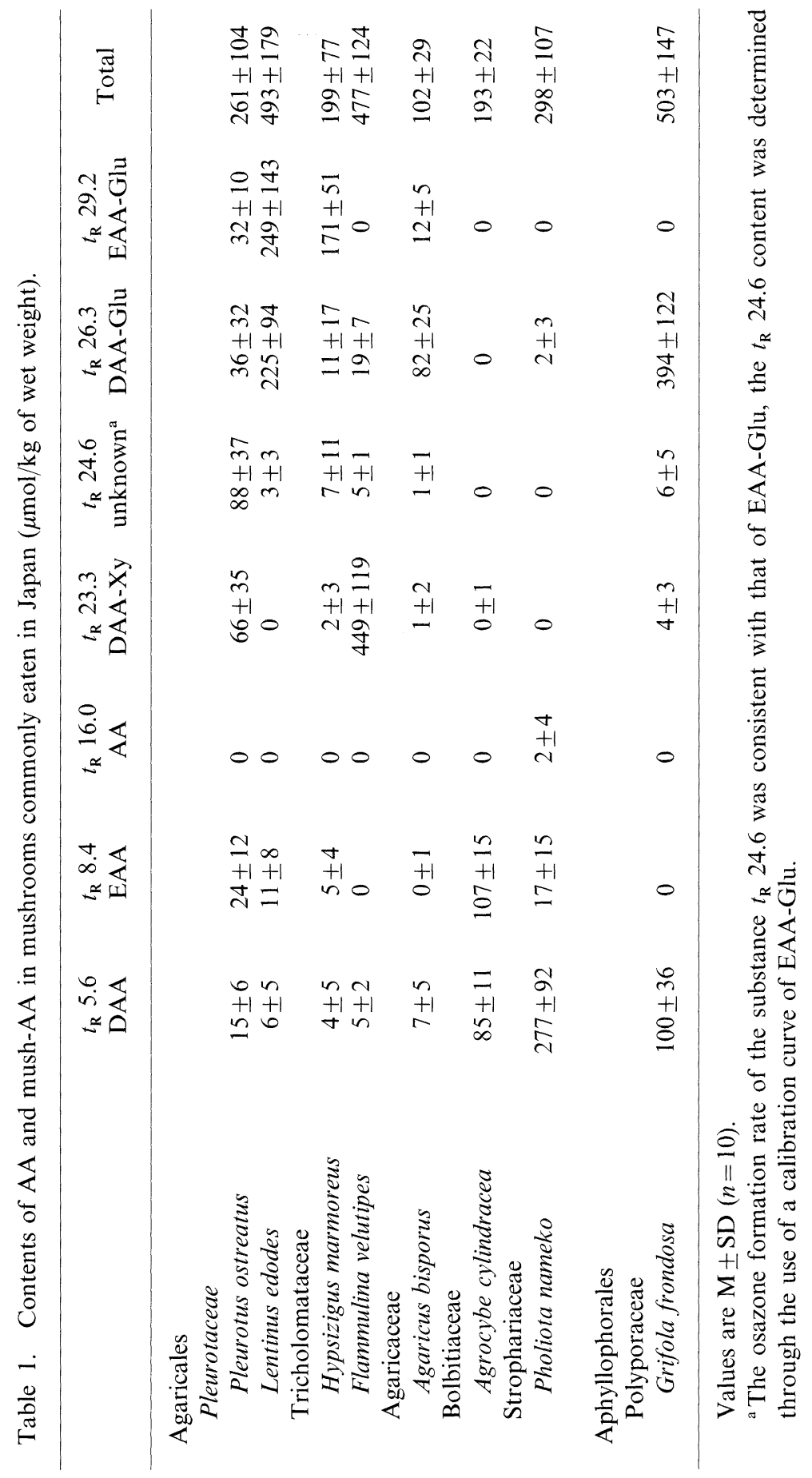


a peak was observed at $t_{\mathrm{R}}$ 24.6. The absorption spectrum agreed with that of AA-osazone, so it is considered to be of an unknown AA analog. In Lentinus edodes, EAA-Glu and DAA-Glu, which are glucose-combined glycosides, constituted $97 \%$ of the total amount, though DAA and EAA exist in small amounts. In Hypsizigus marmoreus, Flammulina velutipes and Agaricus bisporus, single glycosides (EAA-Glu, DAA-Xy and DAA-Glu, respectively) constituted over $80 \%$ of the total amount of constituents. In Agrocybe cylindracea and Pholiota nameko, DAA and EAA were the main constituents, and only a trace of glycosides was found. Particularly in Pholiota nameko, $298 \mu \mathrm{mol}$ of mush-AA per $\mathrm{kg}$ was found, $94 \%$ of which was DAA, and $2 \mu \mathrm{mol} / \mathrm{kg}$ of AA was also found. In Grifola frondosa, DAA-Glu constituted $78 \%$, and DAA, its constituent, constituted $20 \%$ of the total.

Thus, the mush-AA contained in mushrooms is quite varied from one species to another. The total amount of mush-AA contained in mushrooms was between ca. $100-500 \mu \mathrm{mol} / \mathrm{kg}$ (2-9 mg per $100 \mathrm{~g}$, converted to AA). Some constituents with average contents below $10 \mu \mathrm{mol} / \mathrm{kg}$ were not detected pertaining to some individuals; therefore, the constituents listed as zero could be contained in trace amounts below the detection limit. The substance $t_{\mathrm{R}} 24.6$ was contained in other mushrooms as well as Pleurotus ostreatus though it was only a trace amount. None of mushrooms in this study contained D-araboascorbic acid. The total amount of mush-AA obtained by this method is the sum of the reduced and oxidized forms. The measurement of the original oxidized form alone, without oxidation by indophenol during the formation of osazone, showed results within $10 \%$ of the total amount (reduced form + oxidized form) for all mushrooms. Taking oxidation during grinding into account, most mush-AA exists in reduced form in mushrooms.

\section{Structural analysis of the substance $t_{\mathrm{R}} 24.6$}

Based on the absorption spectrum and $t_{\mathrm{R}}$ value, the substance at $t_{\mathrm{R}} 24.6$ is assumed to be assigned to a glycoside of the AA analog. Then, according to the method described in a previous paper (1), this substance was extracted from Pleurotus ostreatus and purified. The purified substance showed reducing power even in acidic conditions. The absorption maximum of the reduced form was $266 \mathrm{~nm}$ at $\mathrm{pH} 5.6$ and $242 \mathrm{~nm}$ at $\mathrm{pH} \mathrm{1.0,} \mathrm{and} \mathrm{no} \mathrm{absorption} \mathrm{was} \mathrm{observed} \mathrm{in} \mathrm{the} \mathrm{oxidized} \mathrm{form.} \mathrm{The}$ purified substance was dissolved in $3 \mathrm{~N} \mathrm{HCl}$ and heated for $15 \mathrm{~min}$ in a boiling water bath for hydrolysis. The hydrolysis product was developed with TLC using Wakogel FM Plate with ethylacetate/acetic acid $/ \mathrm{H}_{2} \mathrm{O}(6: 3: 2, \mathrm{v} / \mathrm{v})$ as the developing solvent; a sugar which can be detected by $p$-anisidine and a reducing substance which decolors indophenol were obtained consequently. The $R f$ value of the sugar $(0.53)$ was the same as that of D-xylose. The substance at $R f 0.53$ was reacted with Bial reagent (12), and its visible absorption spectrum agreed completely with that of pure D-xylose. The absorption maxima of the reducing substance were $266 \mathrm{~nm}$ at $\mathrm{pH} 5.6$ and $243 \mathrm{~nm}$ at $\mathrm{pH} 1.0$. No absorption was observed in the oxidized form. The oxidized form reacted with hydrazine to form osazone, which had an absorption spectrum of the AA-osazone type. The retention time of the osazone in HPLC 
Table 2. Chemical shifts of the substance $t_{\mathrm{R}} 24.6$ and EAA for ${ }^{13} \mathrm{C}-\mathrm{NMR}$ (ppm), and assignment of the signals.

\begin{tabular}{|c|c|c|c|c|c|c|c|}
\hline & $t_{\mathrm{R}} 24.6$ & EAA & $\begin{array}{l}\text { 1-O-methyl- } \alpha \text {-D- } \\
\text { xylopyranose }\end{array}$ & \multicolumn{2}{|c|}{ DAA-Xyb } & \multicolumn{2}{|c|}{ EAA-Glu ${ }^{b, c}$} \\
\hline C-1 & 173.3 & 173.4 & & 173.4 & & $178.1)$ & \\
\hline $\mathrm{C}-2$ & 119.5 & 119.9 & & 119.9 & & 113.5 & \\
\hline C-3 & 155.0 & 154.0 & & 155.6 & & $176.3\}$ & EAA \\
\hline $\mathrm{C}-4$ & 76.7 & 78.5 & & 79.8 & DAA & 78.9 & \\
\hline$C-5$ & 68.1 & 61.6 & & 71.6 & & 66.8 J & \\
\hline C-6 & & & & 15.7 & & & \\
\hline $\mathrm{C}-1^{\prime}$ & 100.5 & & 99.5 & 98.1 & & $98.8]$ & \\
\hline $\mathrm{C}-2^{\prime}$ & 73.5 & & 72.4 & 73.6 & & 72.2 & \\
\hline $\mathrm{C}-3^{\prime}$ & 74.9 & & 74.4 & 75.0 & $\begin{array}{l}\alpha \text {-D- } \\
\text { xylonvranose }\end{array}$ & 73.8 & $\alpha-D-$ \\
\hline C- $4^{\prime}$ & 71.4 & & 70.5 & 71.6 & & $70.4\}$ & glucopyranose \\
\hline$C-5^{\prime}$ & 63.2 & & 61.9 & 63.5 & & 72.5 & \\
\hline$C-6^{\prime}$ & & & & & & 61.3 & \\
\hline
\end{tabular}

${ }^{\mathrm{a}}$ From Ref. 13.

${ }^{\mathrm{b}}$ From Ref. 1.

${ }^{\mathrm{c}} \mathrm{Na}$ salt (C-3: -ONa).

analysis was $8.4 \mathrm{~min}$, which was consistent with that of EAA-osazone. In addition, the number of moles of xylose and the substance considered to be EAA were equal. Based on these results, this substance was presumed to be a glycoside of EAA and xylose. The MS and ${ }^{13} \mathrm{C}$ NMR spectrum of the substance were then measured. A ZAB-HF mass spectrometer (VG Co.) was used for MS, and ionization employed the FAB method. The measurement of positive ions was difficult because the sample contained some contamination, and thus negative ions were measured. A peak considered to be a quasi-molecular ion $\left((\mathbf{M}-\mathbf{H})^{-}\right)$was observed at $m / z=277$. The prehydrolysis substance and pure EAA obtained from EAA-Glu by hydrolysis were then individually dissolved in methanol $\mathrm{d}_{4}$, and their ${ }^{13} \mathrm{C}$ NMR spectra were measured by a GSX400 (JEOL Co.) using tetramethylsilane as the internal standard. Ten peaks for the prehydrolysis substance and five peaks for EAA were observed. These peaks were assigned as listed in Table 2 in accordance with the values of DAA-Xy and EAA-Glu (1). The values of xylopyranose moiety were consistent with those of 1-O-methyl- $\alpha$-D-xylopyranose (13). This identified the substance as 5-O-( $\alpha$-D-xylopyranosyl)-erythroascorbic acid $\left(\mathrm{C}_{10} \mathrm{H}_{14} \mathrm{O}_{9}\right.$; molecular weight: 278$)$ (Fig. 3). Moreover, EAA was dissolved in $0.01 \mathrm{~N} \mathrm{HCl}$ and its rotation angle measured using a SEPA-200 polarimeter (Horiba Co.), resulting in $[\alpha]_{\mathrm{D}}^{20}=$ $-11.97^{\circ}(\mathrm{C}=1)$, which proved to be $\mathrm{D}$-form according to the value in the reference (14). 


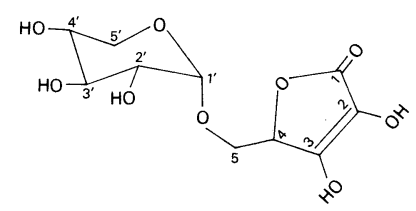

Fig. 3. Proposed structure of the substance $t_{\mathrm{R}}$ 24.6: 5-O-( $\alpha$-D-xylopyranosyl)-Derythroascorbic acid.

\section{DISCUSSION}

This method made it possible to perform the separative determination of AA and mush-AA. The results revealed that AA exists in one species, but that it exists as mush-AA in most mushrooms. Traces of AA were found in Pholiota nameko, and its amount was zero or below the detection limit in other mushrooms. Iijima et al (9) allowed the extract, Lentinus edodes, Flammulina velutipes and Agaricus bisporus to react with hydrazine to form osazones, and compared them through determinations by the colorimetric method of Roe et al (7) and the HPLC method of Kodaka et al (8). They reported that $1.2-4.4 \mathrm{mg}$ of AA per $100 \mathrm{~g}$ was determined for all mushrooms using the colorimetric method, but nothing was determined by HPLC. These results are consistent with the results shown in Table 1. In other words, these mushrooms contain mush-AA and osazones formed by mush-AA are determined by colorimetry, but because they do not contain AA, no peak of AA-osazone is observed using the HPLC method. The types of contained mush-AA vary according to the species and their osazone formation rates vary, so accurate values for total amount cannot be obtained using the colorimetric method. The reason for the lower osazone formation rates of DAA and its glycosides is unclear.

The findings for EAA-Xy in this study revealed that DAA, EAA and their four glycosides, with which glucose or xylose are bonded, exist. These six kinds are considered to be the main AA analogs, but some peaks, presumed to be of AA analog osazones, were observed in the chromatograms. However, no peak could be confirmed because they were so small that their absorption spectra could not be measured. More experimental examples may identify mushrooms containing many unknown AA analogs. The method in this study would also be useful for such a search.

As EAA is found in Candida utilis (15), Neurospora crassa (16), Saccharomyces cerevisiae and Lypomyces starkeyi (6), which are Ascomycetes, and in Basidiomycetes, as has been revealed in this study, EAA can be considered a general substance in fungi. Murakawa et al (15) assumed the synthesis process of EAA in C. utilis to be $\mathrm{D}$-arabinose $\rightarrow \mathrm{D}$-arabono- $\delta$-lactone $\leftrightarrow \mathrm{D}$-arabono- $\gamma$-lactone $\rightarrow \mathrm{D}$-EAA, while Takahashi et al (11) assumed the synthesis process of D-araboascorbic acid in Penicillium to be D-glucose $\rightarrow$ D-gluconate $\rightarrow$ 3-keto-D-gluconate (or D-glucono- $\gamma$ lactone) $\rightarrow$ D-araboascorbic acid. These two studies do not assume the reversal of 
C-1 and C-6, which is observed in the synthesis process of AA in animals. If DAA in mushrooms is synthesized in similar reactions, DAA may be synthesized from deoxy sugar such as L-fucose. As shown in Table 1, the main mush-AA and combination patterns differ significantly depending on the kind of mushrooms, the reason of which will be revealed when the biosynthesis process has been clarified. Incidentally, it is difficult to consider that EAA, DAA and their glycosides are playing different physiological roles in tissues. As the six so-far-determined types of mush-AA display a similar reduction power to that of $\mathrm{AA}$ in vitro, and general metabolism in mushrooms is similar to that of plants, a possible role of mush-AA is the elimination of active oxygen. The amount of mush-AA in mushrooms tends to be lower than that of AA in plant leaves. This is believed to be because mushrooms do not conduct photosynthesis, and are able to thrive under little light. Currently, the biosynthesis process and physiological function of mush-AA can only be hypothesized, and further research needs to be conducted.

I express my deep thanks to Mr. A. Abe, of the Toray Research Center, who assisted me in NMR spectral measurement and structural analysis.

\section{REFERENCES}

1) Okamura M. 1994. Distribution of ascorbic acid analogs and associated glycosides in mushrooms. J Nutr Sci Vitaminol 40: 81-94.

2) Okamura M. 1980. An improved method for determination of L-ascorbic acid and L-dehydroascorbic acid in blood plasma. Clin Chim Acta 103: 259-268.

3) Arakawa N, Otsuka M, Kurata T, Inagaki C. 1981. Separative determination of ascorbic acid and erythorbic acid by high-performance liquid chromatography. J Nutr Sci Vitaminol 27: 1-7.

4) Otsuka M, Kurata T, Suzuki E, Arakawa N, Inagaki C. 1981. Separative determination of ascorbic acid and erythorbic acid in animal tissues by high-performance liquid chromatography. J Nutr Sci Vitaminol 27: 9-15.

5) Kasai T, Tsujimura M, Hukuda T. 1982. Synthesis and vitamin C activity of L-threo-hexo-2-enaro-1,4-lactone. Vitamins (Japan) 56: 117-131.

6) Nick JA, Leung CT, Loewus FA. 1986. Isolation and identification of erythroascorbic acid in Saccharomyces cerevisiae and Lypomyces starkeyi. Plant Sci 46: 181-187.

7) Roe JH, Kuether CA. 1943. The determination of ascorbic acid in whole blood and urine through the 2,4-dinitrophenylhydrazine derivative of dehydroascorbic acid. $J$ Biol Chem 147: 399-407.

8) Kodaka K, Inagaki S, Ujiie T, Ueno T, Suda H. 1985. Determination of total vitamin $\mathrm{C}$ in foodstuffs by high-performance liquid chromatography. Vitamins (Japan) 59: $451-455$.

9) Iijima T, Baba O, Kawata T, Ueno T, Tadokoro T, Maekawa A. 1989. Comparison of total ascorbic acid values in food by 2,4-dinitrophenylhydrazine method, $\alpha, \alpha^{\prime}$-dipyridyl method and high-performance liquid chromatography. Vitamins (Japan) 63: 497-502.

10) Tsujimura M, Higasa S, Kasai T. 1996. Quantitative determination of total vitamin C in foods by HPLC using 2,4-dinitrophenylhydrazine as the internal standard. Vitamins 
(Japan) 70: 241-248.

11) Takahashi T, Mitsumoto M, Kayamori H. 1960. Production of D-araboascorbic acid by Penicillium. Nature 188: 411-412.

12) Mejbaum W. 1939. Über die Bestimmung kleiner Pentosemengen, insbesondre in Derivaten der Adenylsäure. Z Physiol Chem 258: 117-120.

13) Stothers JB. 1972. Carbon 13-NMR Spectroscopy, p 461-462. Academic Press, New York.

14) Reichstein T. 1938. 3-Keto-D-pentonic acid lactone and process for the manufacture of the same. USA Patent 2,140,480.

15) Murakawa S, Sano S, Yamashita A, Takahashi T. 1977. Biosynthesis of D-erythroascorbic acid by Candida. Agric Biol Chem 41: 1799-1800.

16) Dumbrava V, Pall ML. 1987. Control of nucleotide and erythroascorbic acid pools by cyclic AMP in Neurospora crassa. Biochim Biophys Acta 926: 331-338. 